\title{
Effective immunosuppression with dexamethasone phosphate in the Galleria mellonella larva infection model resulting in enhanced virulence of Escherichia coli and Klebsiella pneumoniae
}

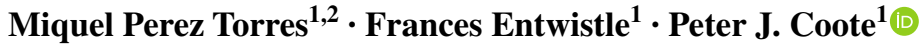

Received: 19 November 2015 / Accepted: 11 February 2016 / Published online: 26 February 2016

(C) The Author(s) 2016. This article is published with open access at Springerlink.com

\begin{abstract}
The aim was to evaluate whether immunosuppression with dexamethasone 21-phosphate could be applied to the Galleria mellonella in vivo infection model. Characterised clinical isolates of Escherichia coli or Klebsiella pneumoniae were employed, and G. mellonella larvae were infected with increasing doses of each strain to investigate virulence in vivo. Virulence was then compared with larvae exposed to increasing doses of dexamethasone 21-phosphate. The effect of dexamethasone 21-phosphate on larval haemocyte phagocytosis in vitro was determined via fluorescence microscopy and a burden assay measured the growth of infecting bacteria inside the larvae. Finally, the effect of dexamethasone 21-phosphate treatment on the efficacy of ceftazidime after infection was also noted. The pathogenicity of K. pneumoniae or E. coli in G. mellonella larvae was dependent on high inoculum numbers such that virulence could not be attributed specifically to infection by live bacteria but also to factors associated with dead cells. Thus, for these strains, G. mellonella larvae do not constitute an ideal infection model. Treatment of larvae with dexamethasone 21-phosphate enhanced the lethality induced by infection with $E$. coli or $K$. pneumoniae in a dose- and inoculum size-dependent manner. This correlated with proliferation of bacteria in the larvae that could be attributed to dexamethasone inhibiting haemocyte phagocytosis and acting as an immunosuppressant. Notably, prior exposure to
\end{abstract}

Peter J. Coote

pjc5@st-andrews.ac.uk

1 Biomedical Sciences Research Complex, School of Biology, University of St Andrews, The North Haugh, St Andrews, Fife KY16 9ST, UK

2 Present Address: Department of Microbiology, Facultad de Biología, University of Barcelona, Diagonal, 643, 08028 Barcelona, Spain dexamethasone 21-phosphate reduced the efficacy of ceftazidime in vivo. In conclusion, demonstration of an effective immunosuppressant regimen can improve the specificity and broaden the applications of the G. mellonella model to address key questions regarding infection.

Keywords Insect infection model - Antibacterial · Antimicrobial · Ceftazidime · Pathogenicity ·

Glucocorticoid anti-inflammatory

\section{Introduction}

Globally, multidrug-resistant (MDR) Gram-negative bacteria are a major cause of hospital-acquired infections and are considered an urgent public health threat [1]. The majority of these infections are either pneumonia, bloodstream or urinary tract infections and are associated with invasive medical devices or surgical procedures and effect patients that are already debilitated or immunocompromised (reviewed in [2]). With increasing incidence of resistance to carbapenems, the antibiotics of 'last-resort', only a limited number of antibiotic treatments are available for some MDR bacteria [3] resulting in high mortality rates [4].

In the USA, the most common MDR bacteria associated with hospital-acquired infections are Klebsiella pneumoniae and Escherichia coli [1]. MDR strains of these organisms initially acquired a variety of extended-spectrum $\beta$-lactamases (ESBLs) that rendered them resistant to cephalosporin antibiotics resulting in increased use of carbapenems. Subsequently, this selected for the acquisition of carbapenemases rendering strains resistant to this class of drugs also. In the UK, the first strain of $K$. pneumoniae with resistance to carbapenems due to expression of the K. pneumoniae carbapenemase 3 enzyme (KPC-3) 
was reported in 2007 [5]. Also in the UK, concern was first raised in 2003 that MDR E. coli expressing the CTX-M-15 ESBL were increasingly being identified in patients with community-acquired urinary tract infections that had no previous history of hospitalisation [6]. This spread of MDR pathogens into general practice with few treatment options represents a serious threat to public health.

Thus, there is clear need to research and develop new antibiotics and treatment regimens to combat MDR Gramnegative infections. To facilitate this process, the use of animal models to measure efficacy of potential new treatments is an indispensable part of the drug discovery pipeline. Presently, the majority of novel antimicrobials that are initially identified in vitro are then tested for efficacy in vivo using murine infection models. The problems associated with employing mammalian infection models include ethical issues, high costs and their impracticality for large-scale screening of efficacy. Thus, researchers are increasingly using alternative infection models such as invertebrates to circumvent these issues.

Larvae of the greater wax moth Galleria mellonella have been employed as an in vivo model in many investigations of bacterial pathogenicity or efficacy of antibiotic treatments (reviewed in [7]). Advantages of G. mellonella include: fewer ethical concerns, low cost, precise inoculation of bacteria and/or dosing of drugs, ability to incubate larvae at human body temperature and ease of use with regard to high-throughput screening of novel treatments in vivo. Disadvantages include the reduced complexity of the invertebrate immune system compared to mammals due to the absence of adaptive immunity, the inability to study long-term, chronic infections and the exclusion of pathogens that do not display virulence in the larvae.

Galleria mellonella larvae have been used to characterise pathogenicity and virulence of $K$. pneumoniae clinical isolates and strains with mutations in known virulence factor genes [8-10]. Furthermore, G. mellonella was used to quantify the efficacy of antibiotics against planktonic or biofilm cultures of MDR strains of $K$. pneumoniae [11]. Similar studies with pathogenic $E$. coli have also been undertaken with $G$. mellonella that have correlated lethality with specific virulence factors in uropathogenic $[12,13]$ and enteropathogenic isolates [14]. Also, G. mellonella was comparable to a murine model for measuring virulence of a range of E. coli clinical isolates [15]. Notably, assessment of the inoculum size of K. pneumoniae or E. coli that was required to induce significant lethality in G. mellonella reveals huge variability between bacterial strains but also that the minimum number of bacteria required to cause significant larval death, even with the most virulent strains tested, was much higher with $K$. pneumoniae and $E$. coli compared with that required for another Gram-negative pathogen Pseudomonas aeruginosa [16, 17].
In this study, the effect of treating G. mellonella larvae with a water-soluble derivative of the immunosuppressant drug dexamethasone on subsequent susceptibility to infection by $K$. pneumoniae and $E$. coli was evaluated. The aims of the study were: (1) to decrease the relatively high inoculum of $K$. pneumoniae or $E$. coli cells required to induce a lethal infection in G. mellonella larvae to explore whether immunosuppression could be employed as a viable route to expand use of this model to pathogens that do not display virulence or induce acute infections in the larvae; (2) to mimic the immunosuppressed state that is often a significant factor with human patients that suffer from hospital-acquired infections by K. pneumoniae and E. coli; and finally (3) to assess the effect of immunosuppression on the efficacy of antibiotic treatment for K. pneumoniae or E. coli infection in vivo.

\section{Materials and methods}

\section{Bacteria and growth media}

Bacteria were obtained from the National Collection of Type Cultures (NCTC) (http://www.hpacultures.org.uk/ collections/nctc.jsp). The $K$. pneumoniae strains were NCTC9633 (type strain) and NCTC13438 (a MDR isolate possessing the KPC-3 carbapenemase; [5]), and the E. coli strains were NCTC12241 (a control strain for antibiotic sensitivity testing) and NCTC13353 (an isolate possessing the CTX-M-15 ESBL; [6]). All of these strains are clinical isolates. Strains were cultured overnight in Mueller-Hinton Broth (MHB; Merck, Darmstadt, Germany) at $37{ }^{\circ} \mathrm{C}$ with shaking to prepare inocula for in vivo infections.

\section{Reagents and G. mellonella larvae}

Unless stated, all chemicals and ceftazidime hydrate were purchased from Sigma-Aldrich Ltd (Dorset, UK). Stock solutions and sub-stocks of all drugs for use in experiments were made in sterile deionised water. G. mellonella larvae were purchased from UK Waxworms Ltd (Sheffield, UK).

\section{Galleria mellonella infection model and treatment with dexamethasone}

This was carried out as previously described [17]. Briefly, groups of 15 larvae were infected with variable inoculum sizes of $K$. pneumoniae or E. coli cells and incubated at $37{ }^{\circ} \mathrm{C}$. Larval survival was determined every $24 \mathrm{~h}$ for $96 \mathrm{~h}$ in total. Precise numbers of bacterial cells inoculated were determined by serial dilution in MHB and plating on Nutrient Agar (NA; Merck, Darmstadt, Germany) prior to counting colonies after overnight incubation of plates at $37{ }^{\circ} \mathrm{C}$. Each experiment was repeated at least twice using 
larvae from different batches. The data from these replicate experiments were then pooled, and survival data were plotted using the Kaplan-Meier method and comparisons made between groups using the log-rank test. In all comparisons to the negative control, it was the uninfected control (rather than the unmanipulated control) that was used. In all tests, $p \leq 0.05$ was considered significant and Holm's correction was applied to account for multiple comparisons [18]. Heat-killed $\left(98^{\circ} \mathrm{C}, 15 \mathrm{~min}\right.$ ) inocula at the highest inoculum sizes used were also injected as controls. Heat-killing was verified by plating the heated inocula on NA. To identify any secreted bacterial virulence factors both heat-killed and live inocula were sterile-filtered using Millex $0.22 \mu \mathrm{M}$ syringe-driven filters (Millipore Ltd, Tullagren, Co. Cork, Ireland) and the filtrate injected into the larvae.

The effect of exposure to dexamethasone was tested by injecting dexamethasone 21-phosphate disodium $(50 \mathrm{mg} /$ $\mathrm{mL}$ stock solution in $\mathrm{H}_{2} \mathrm{O}$ ) for variable time periods (10 min, 2, 4, 6 and $8 \mathrm{~h}$ ) or at variable doses ( 80 or $200 \mu \mathrm{g}$ per larva) prior to infection of larvae with bacteria.

For experiments involving a single dose of ceftazidime, the antibiotics were administered $2 \mathrm{~h}$ post-infection (p.i).

\section{Galleria mellonella haemolymph burden}

Groups of 40 larvae were treated with $200 \mu \mathrm{g}$ per larva of dexamethasone 21-phosphate disodium prior to infection with known numbers of $K$. pneumoniae or $E$. coli cells and incubation at $37{ }^{\circ} \mathrm{C}$. After $5 \mathrm{~h}$ incubation, and every $24 \mathrm{~h}$ thereafter, five larvae were selected at random from each treatment group and tested for haemolymph burden exactly as previously described [19].

\section{Phagocytosis assays}

The method was adapted from Mandato et al. [20]. Larvae were chilled at $4{ }^{\circ} \mathrm{C}$ for $15 \mathrm{~min}$ prior to injection with $75 \mu \mathrm{L}$ of Galleria saline (GS; $186 \mathrm{mM} \mathrm{NaCl}, 13 \mathrm{mM} \mathrm{KCl}$, $10 \mathrm{mM}$ HEPES, $1 \mathrm{mM} \mathrm{NaHCO}{ }_{3}, 2 \mathrm{mM} \mathrm{CaCl} 2 ; \mathrm{pH} \mathrm{6.8)}$ saturated with phenylthiol urea (PTU). Haemolymph was collected from 5 larvae per assayed treatment via amputation of the terminal thoracic prolegs. The haemolymph was centrifuged $5 \mathrm{~min}$ at $500 \mathrm{~g}$ and the haemocytes resuspended in GIM (Grace's Insect Medium) saturated with PTU and dexamethasone 21-phosphate disodium at $5 \mathrm{mg} / \mathrm{mL}$ or water for the untreated control. Haemocyte viability was measured by counting haemocytes that exclude Trypan blue $(0.02 \%$ in PBS) using a haemocytometer exactly as described previously [20]. $250 \mu \mathrm{L}$ of this suspension was placed in separate poly (2-hydroxyethyl methacrylate)-coated wells in 24-well culture plates $\left(\right.$ Cellstar $^{\circledR}$, Greiner bio-one, Frickenhausen, Germany). After $15 \mathrm{~min}$, inactivated fluorescein (FITC)labelled E. coli K12 Bioparticles ${ }^{\circledR}$ (ThermoFisher Scientific,
Waltham, MA, USA), $1.0 \times 10^{8}$ lyophilised cells per $\mathrm{mL}$ resuspended in GS + PTU, were added and incubated at $37{ }^{\circ} \mathrm{C}$ for 1 and $2 \mathrm{~h}$ with gentle agitation. Haemocytes were quantified using a haemocytometer, and the number of fluorescent bacteria added was calculated such that that the proportion of bacteria to haemocytes was approximately 50:1. The assay was stopped by addition of $1 \mathrm{~mL}$ of $3.7 \%$ formaldehyde in GS. The fixed haemocytes were then pelleted at $500 \mathrm{~g}$ for $4 \mathrm{~min}$ and washed with GS to remove excess fluorescent bacteria prior to a 1:1 dilution in glycerol and dispensing on glass microscope slides. Haemocytes and fluorescent $E$. coli were viewed at $40 \times$ magnification using a Delta Vision microscope (Applied Precision, Issaquah, Wash., USA) using differential interference contrast and filters for FITC (excitation $490 \mathrm{~nm} / \mathrm{emission} 528 \mathrm{~nm}$ ). Composite images were captured of optical slices through each haemocyte, and internalised fluorescent bacteria were counted. For each time point tested, phagocytosed $E$. coli from at least 30 haemocytes, from at least two replicate experiments, were counted.

\section{Results}

The pathogenicity of some clinical isolates of $K$. pneumoniae or E. coli in G. mellonella larvae is dependent on high inoculum numbers and cannot be attributed specifically to infection by live bacteria

The effect of infection with $K$. pneumoniae and E. coli strains on survival of G. mellonella is shown in Fig. 1. There was variation between all the strains in the inoculum size required to kill the majority of the larval population, but, supporting previous studies [8-15], the inoculum size required to kill the majority of infected larvae $24 \mathrm{~h}$ post-infection was comparable to that reported for different strains of the same organisms. For all bacterial strains, larval survival over $96 \mathrm{~h}$ was reduced in a dose-dependent manner as the inoculum numbers increased. Notably, infection with heat-killed inocula of each strain at the highest dose tested induced variable lethality. For example, infection with either heat-killed K. pneumoniae NCTC9633 $\left(4.0 \times 10^{6}\right.$ per larva) or E. coli NCTC12241 $\left(1.1 \times 10^{6}\right.$ per larva) killed approximately $10 \%$ of the population after $96 \mathrm{~h}$, whereas heat-killed $K$. pneumoniae NCTC13438 $\left(1.5 \times 10^{5}\right.$ per larva) or E. coli NCTC13353 $\left(7.8 \times 10^{6}\right.$ per larva) killed approximately 50 and $40 \%$, respectively, after 96 h (Fig. 1). To investigate this further, identical heat-killed inocula of $K$. pneumoniae NCTC13438 and E. coli $\mathrm{NCTC} 13353$ that were subsequently filter-sterilised and then injected into larvae had no detrimental effect on larval survival (data not shown; $p>0.05$, log-rank test). Similarly, viable inocula of both strains that were filter-sterilised prior to injection also 

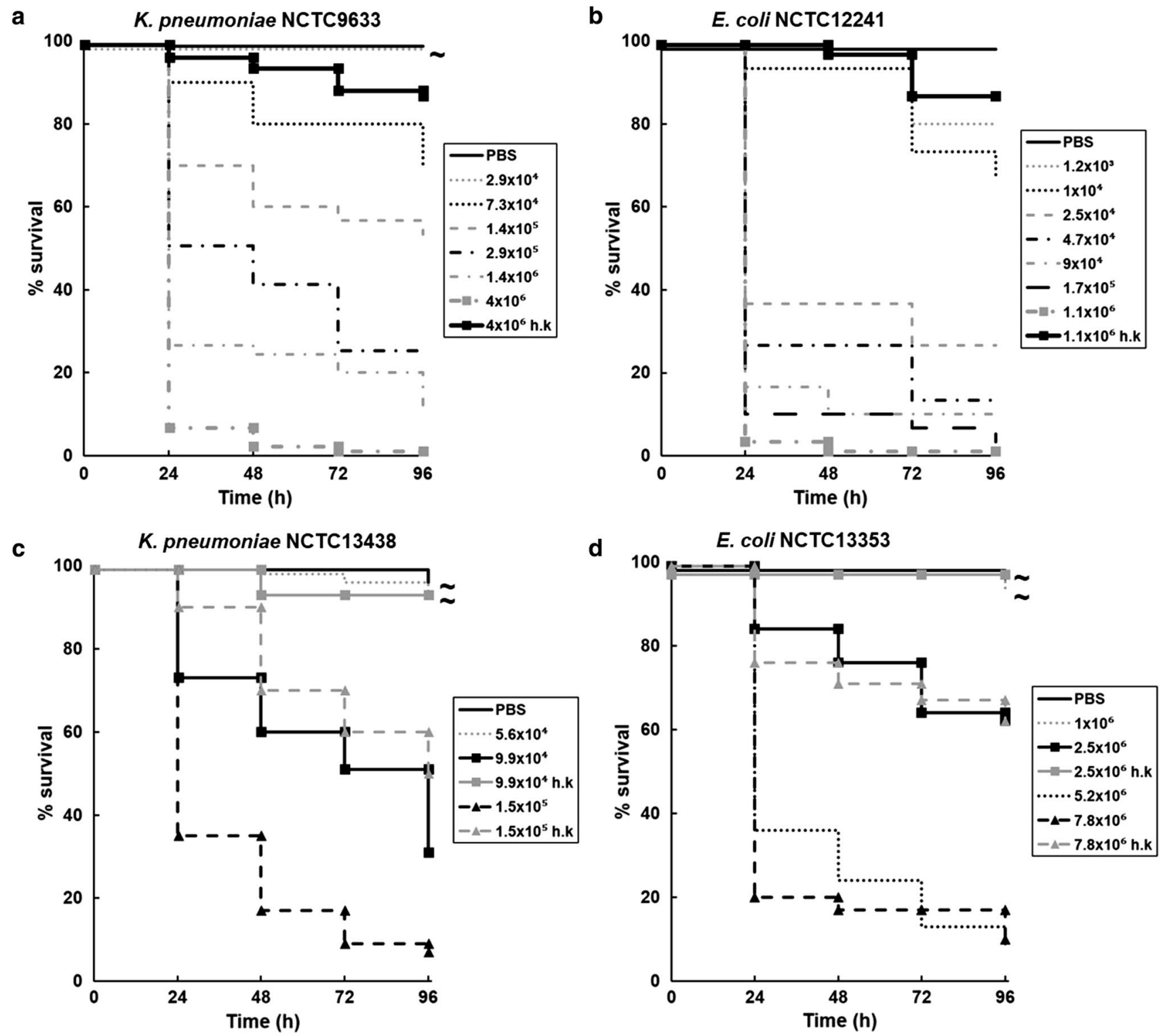

Fig. 1 Effect of increasing inoculum dose of live $K$. pneumoniae NCTC9633 (a) or NCTC13438 (c) and E. coli NCTC12241 (b) or NCTC13353 (d) on the survival of G. mellonella larvae during incubation at $37{ }^{\circ} \mathrm{C}$ for $96 \mathrm{~h}$. Numbers in the legend indicate the inoculum size in cells per larva. For all strains, the effect of heat-killed (h.k.) bacterial inocula is also shown. No significant mortality was observed

had no detrimental effect on larval survival (data not shown; $p>0.05$, log-rank test). These results indicate that larval death induced by the heat-killed cells was due to the presence of these dead cells and not because of any secreted, or released, virulence factors present in the medium.

In conclusion, at the high inoculum levels of K. pneumoniae NCTC13438 and E. coli NCTC13353 required to kill greater than $90 \%$ of the larval population, bacterial pathogenesis cannot be attributed solely to infection by live bacteria because some toxicity is induced simply by the in an unmanipulated group (data not shown) or in the uninfected group sham-infected with sterile PBS. With all treatment groups not labelled with tilde, survival was significantly reduced compared to the group sham-infected with PBS $(p<0.05$, log-rank test with Holm correction for multiple comparisons); $n=45$ (pooled from replicate experiments)

presence of an unknown virulence factor(s) associated with dead cells. Thus, for these particular bacterial strains, $G$. mellonella larvae do not constitute an ideal infection model.

Treatment with dexamethasone 21-phosphate inhibits phagocytosis and sensitises $G$. mellonella larvae to infection by $K$. pneumoniae or $E$. coli

Dexamethasone is a glucocorticoid anti-inflammatory drug that is used extensively in human medicine. Research has 

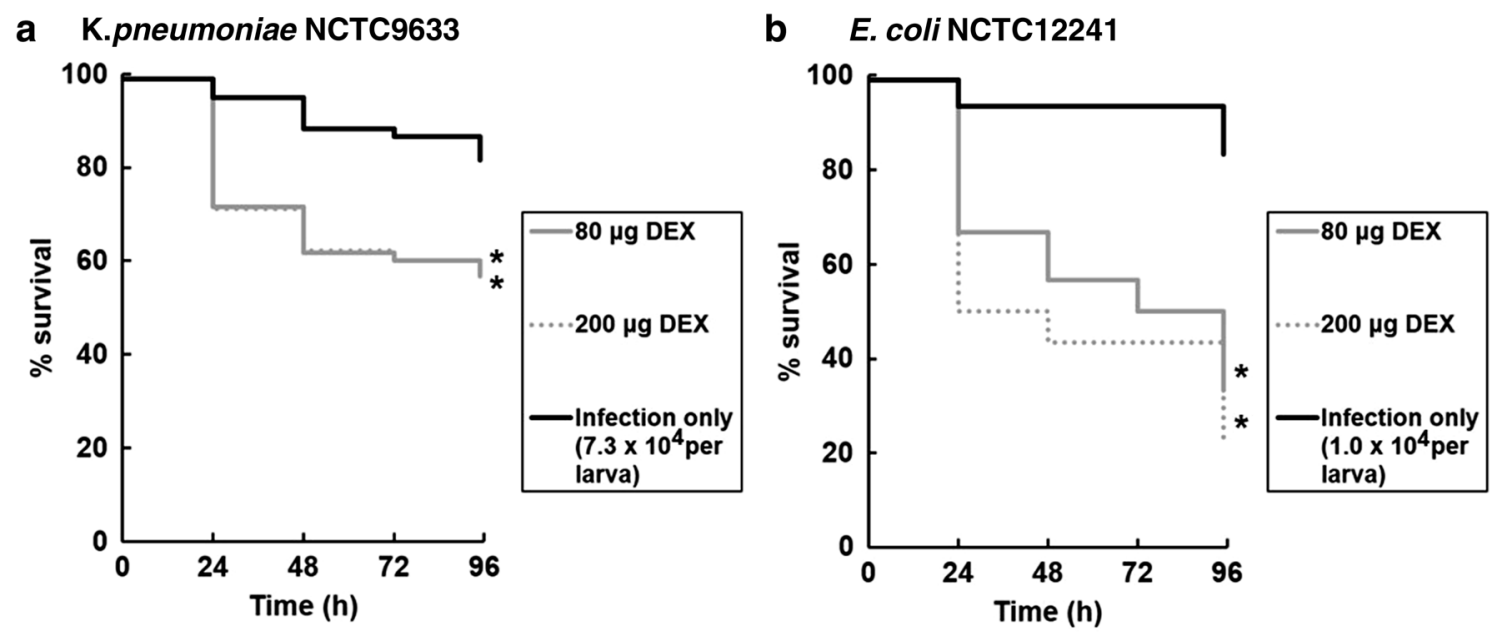

C K.pneumoniae NCTC13438

d E. coli NCTC13353
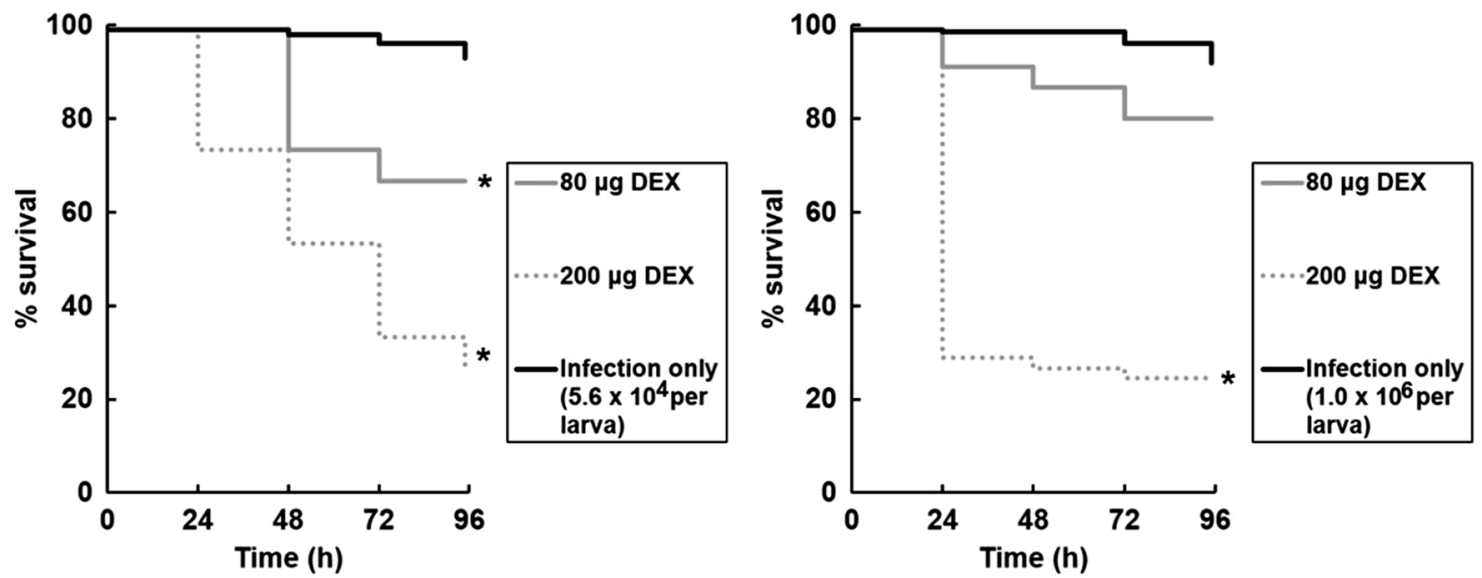

Fig. 2 Effect of an increasing dose of dexamethasone 21-phosphate (DEX; 80 or $200 \mu \mathrm{g}$ per larva) on the survival of G. mellonella larvae infected with $K$. pneumoniae NCTC9633 (a) or NCTC13438 (c) and $E$. coli $\mathrm{NCTC} 12241$ (b) or NCTC13353 (d) during incubation at $37^{\circ} \mathrm{C}$ for $96 \mathrm{~h}$. Numbers in the legend indicate the bacterial inoculum size in cells per larva. The control treatment groups labelled 'infection only' were injected with sterile water in place of dexamethasone

shown that dexamethasone also has immunomodulatory activity in G. mellonella larvae by inhibiting phagocytosis in vitro and prophenoloxidase activation in vivo [20]. Mandato et al. [20] administered water insoluble dexamethasone dissolved in ethanol to larvae. Here, the effect of dexamethasone 21-phosphate, the soluble form of the drug used in human medicine, was measured to determine whether it too could induce immunosuppression in $G$. mellonella larvae and whether it could be used to reduce the inoculum size of $E$. coli or $K$. pneumoniae required to cause significant lethality, thus improving the specificity of this infection model for these organisms.

Trial experiments determined the optimal exposure time and doses of dexamethasone 21-phosphate (data not shown). The effect of a 10-min exposure to 80 and

21-phosphate. Dexamethasone 21-phosphate or sterile water was administered to the larvae $10 \mathrm{~min}$ prior to infection with live bacteria. Asterisk indicates a group with significantly reduced survival compared to the control treated with water $(p<0.05, \log$-rank test with Holm correction for multiple comparisons); $n=30$ (pooled from duplicate experiments)

$200 \mu \mathrm{g}$ per larva doses of dexamethasone 21-phosphate prior to bacterial infection with E. coli or K. pneumoniae on survival of $G$. mellonella larvae is shown in Fig. 2. For each bacterial strain tested, deliberately small inoculum numbers were selected for infection to easily identify any increases in lethality that occurred due to exposure to dexamethasone. For all strains, prior treatment with dexamethasone, compared with sterile water, resulted in significantly increased lethality of infection of an identical inoculum. The increase in mortality also occurred in a dose-dependent manner for all strains, apart from infection with $K$. pneumoniae NCTC9633, where the higher dose of $200 \mu \mathrm{g}$ per larva dexamethasone induced no additional larval mortality over that induced by the $80 \mu \mathrm{g}$ per larva dose. 
Fig. 3 Effect of pre-treatment with dexamethasone 21-phosphate (DEX; $200 \mu \mathrm{g}$ per larva) and subsequent infection with increasing inoculum sizes of K. pneumoniae NCTC9633 (a) or NCTC13438 (c) and E. coli $\mathrm{NCTC} 12241$ (b) or NCTC13353 (d) on the survival of $G$. mellonella larvae after $96 \mathrm{~h}$ at $37^{\circ} \mathrm{C}$. Groups treated with dexamethasone 21-phosphate were compared to controls treated with sterile water. Dexamethasone 21-phosphate or water was administered to the larvae 10 min prior to infection with live bacteria. Asterisk indicates a group with significantly reduced survival compared to the control treated with water $(p<0.05$, log-rank test with Holm correction for multiple comparisons); $n=30$ (pooled from duplicate experiments). \pm Standard deviation error bars are shown for each treatment group
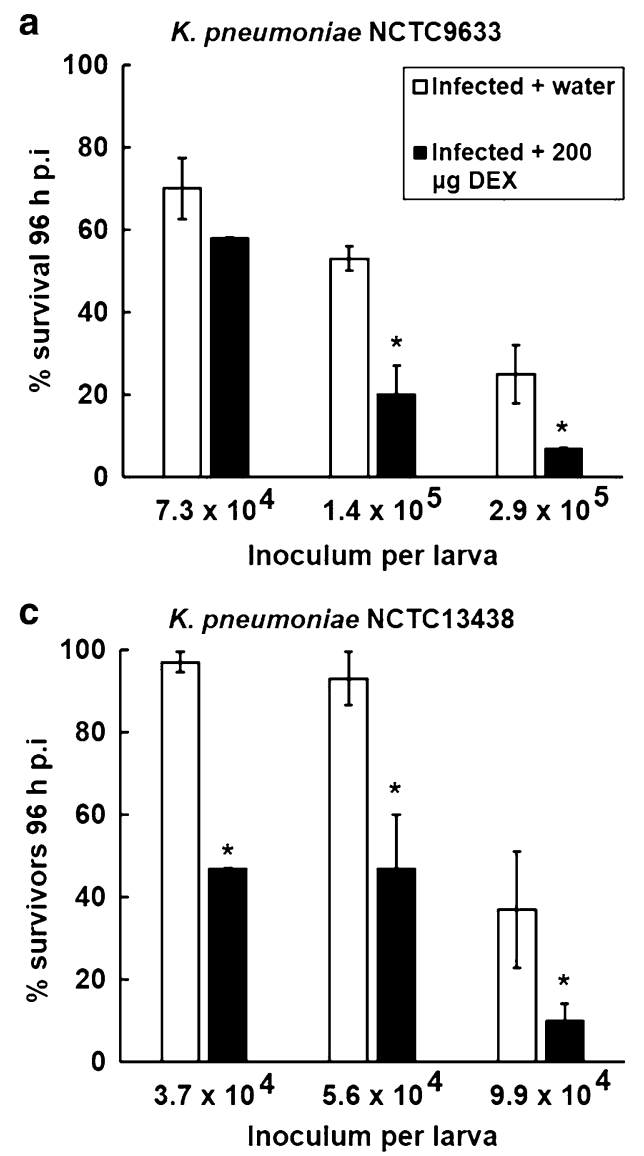
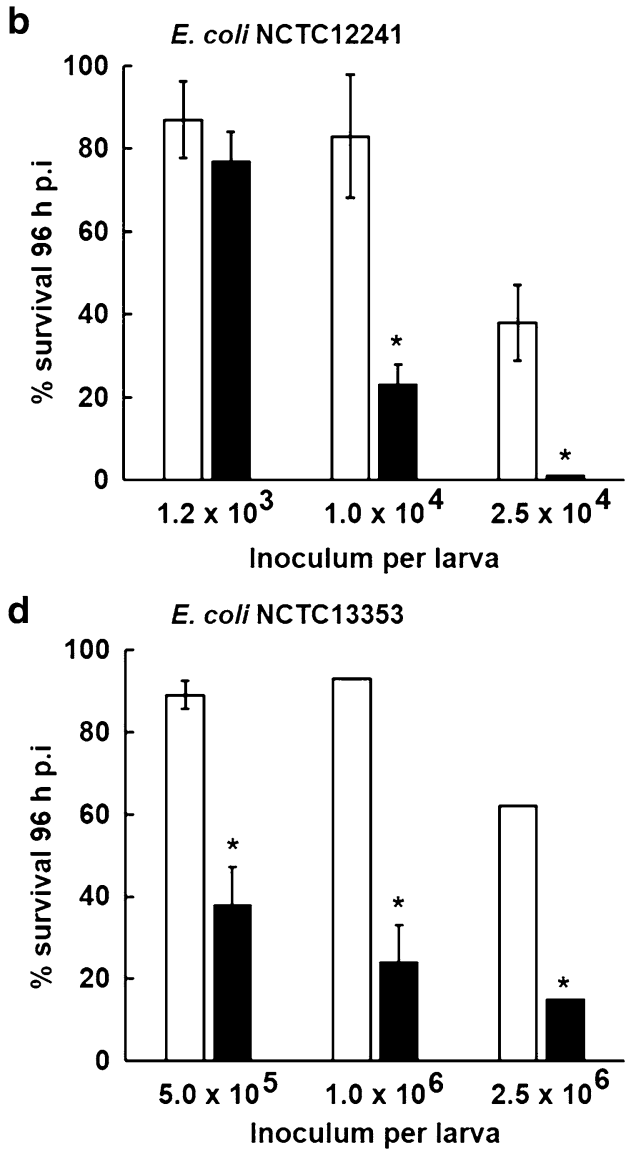

Following this, experiments determined the effect of increasing the inoculum size of infecting bacteria after prior treatment $(10 \mathrm{~min})$ with a fixed dose of dexamethasone 21-phosphate (Fig. 3). As expected, larval groups exposed to water, or dexamethasone (200 $\mu \mathrm{g}$ per larva), for $10 \mathrm{~min}$ prior to infection showed significantly increased mortality in a dose-dependent manner as the inoculum size of all strains increased. Notably, this inoculum-sizedependent lethality was significantly enhanced in larvae that had been pre-treated with dexamethasone $(200 \mu \mathrm{g}$ per larva) for 10 min prior to infection compared to the control groups treated with sterile water.

Importantly, at the concentrations tested, dexamethasone 21-phosphate treatment alone had no detrimental effects on G. mellonella larvae, thus ruling out the possibility that the enhanced lethality of infection induced by treatment with the compound could be explained by a non-specific toxic effect (data not shown; $p>0.05$, log-rank test).

To confirm that the enhanced lethality of infection induced by exposure to dexamethasone 21-phosphate was due to a specific immunosuppressive effect, and whether the compound inhibits phagocytosis in G. mellonella similar to pure dexamethasone [20], phagocytosis assays were carried out. The effect of a 1- or $2 \mathrm{~h}$ incubation with $5 \mathrm{mg} /$ $\mathrm{mL}$ dexamethasone 21-phosphate on phagocytosis in vitro of FITC-labelled E. coli by G. mellonella haemocytes is shown in Fig. 4. This concentration was selected after trial experiments with a range of concentrations (data not shown).

Figure 4a shows a representative image of a single $G$. mellonella haemocyte with phagocytosed FITC-labelled E. coli $\mathrm{K} 12$ taken after $1 \mathrm{~h}$ incubation at $37^{\circ} \mathrm{C}$. Bacteria are visible inside the haemocyte and were counted after studying images of optical slices through the haemocyte. In control experiments (treated with water), the proportion of haemocytes that contained phagocytosed E. coli was approximately $25 \%$ after $1 \mathrm{~h}$ and $30 \%$ after $2 \mathrm{~h}$ (Fig. 4b). Exposure to $5 \mathrm{mg} / \mathrm{mL}$ dexamethasone 21-phosphate reduced this to approximately 12 and $15 \%$ after 1 and $2 \mathrm{~h}$, respectively (Fig. $4 \mathrm{~b}$ ). The observed inhibition of phagocytosis was not due to a toxic effect of dexamethasone 21-phosphate because measurement of haemocyte viability revealed no significant detrimental effect of exposure to the compound under the conditions tested (data not shown).

The effect of immunosuppressive treatment with dexamethasone 21-phosphate on the proliferation of bacteria in $G$. mellonella larvae over $96 \mathrm{~h}$ was measured via a bacterial burden assay (Fig. 5). Inoculum sizes of $E$. coli NCTC13353 $\left(1.0 \times 10^{6}\right.$ cells per larva) and K. pneumoniae 

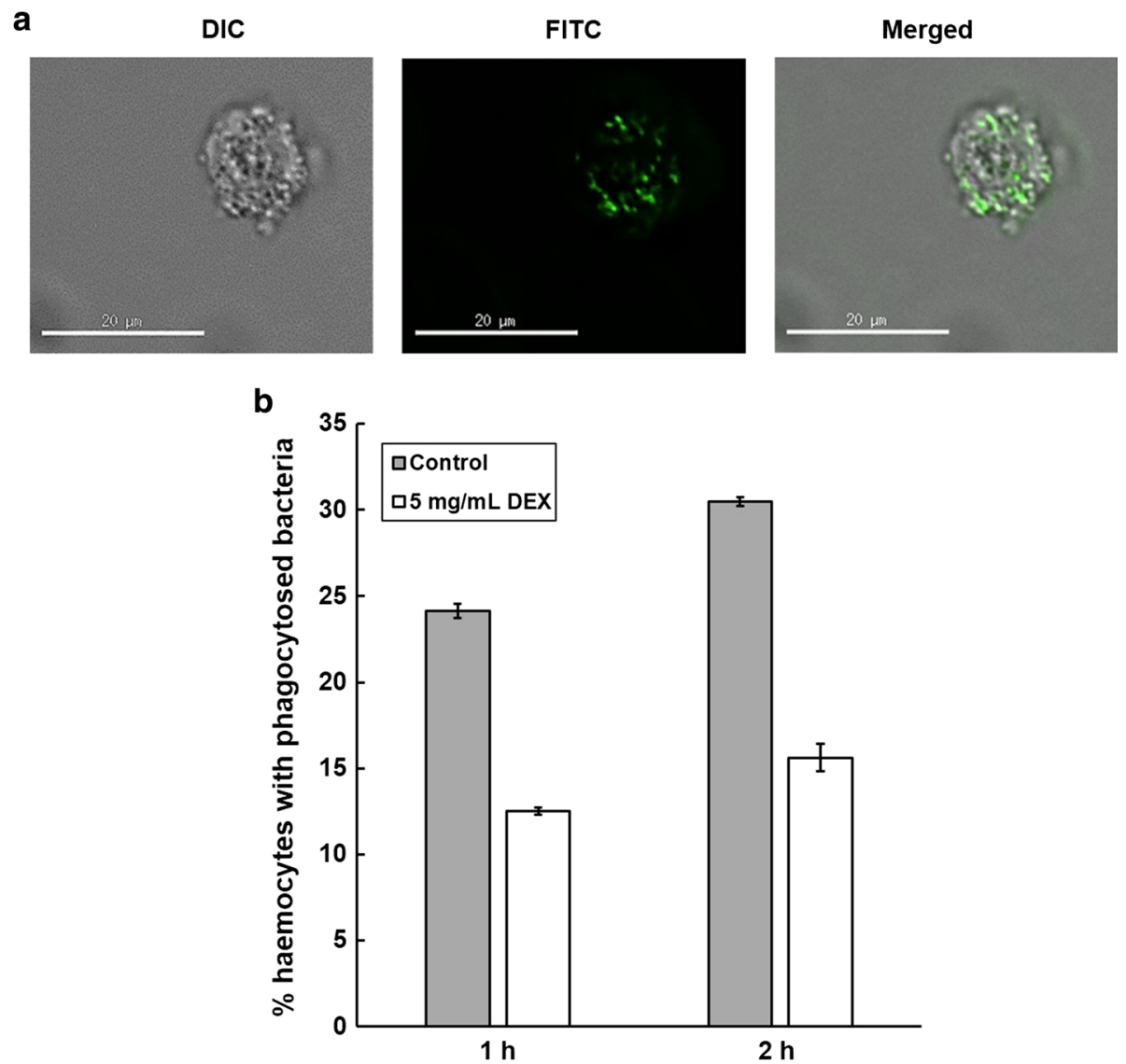

Fig. 4 a Representative image of a single G. mellonella haemocyte after $1 \mathrm{~h}$ incubation at $37{ }^{\circ} \mathrm{C}$ with inactivated FITC-labelled $E$. coli K12. Images are shown with differential interference contrast (DIC), filters for FITC (excitation $490 \mathrm{~nm} /$ emission $528 \mathrm{~nm}$ ) and a merged DIC/FITC image. The merged image represents a central $0.2 \mu \mathrm{M}$ optical slice through the haemocyte allowing precise counting of internalised bacteria. Haemocytes and fluorescent E. coli were viewed at $40 \times$ magnification. b Effect of a 1- or $2 \mathrm{~h}$ incubation at $37{ }^{\circ} \mathrm{C}$ with dexamethasone 21-phosphate (DEX; $5 \mathrm{mg} / \mathrm{mL}$ ) on the phagocytosis

NCTC13438 $\left(5.6 \times 10^{4}\right.$ cells per larva $)$ were chosen that induced large differences in levels of mortality in populations that were pre-treated with water compared to $200 \mu \mathrm{g}$ per larva of dexamethasone 21-phosphate (Fig. 3). In control populations of larvae, infected with either the E. coli or the $K$. pneumoniae strain after pre-treatment with water, the bacterial population size did not increase over the $96 \mathrm{~h}$ duration of the experiment (Fig. 5). This implies that the innate immune system of the larvae prevents the bacteria from proliferating and reflects the low levels of mortality observed under these conditions (Fig. 3). In contrast, pre-treatment with 80 or $200 \mu \mathrm{g}$ per larva of dexamethasone prior to infection resulted in significant increases in bacterial numbers, of either the E. coli or the K. pneumoniae strain, inside the larvae after just $24 \mathrm{~h}$ (Fig. 5). Post-24 h, there is some fluctuation in total bacterial numbers detected that may be due to the random sampling of the five larvae from the total population for each time point. Nonetheless, this proliferation of of inactivated fluorescein (FITC)-labelled E. coli K12 by purified haemocytes from G. mellonella larvae. Haemocytes and fluorescent $E$. coli were viewed using differential interference contrast or fluorescence microscopy at $40 \times$ magnification, and bacteria inside the haemocytes were counted. For each time point tested, phagocytosed E. coli from at least 30 haemocytes, from at least two replicate experiments, were counted per slide. \pm SE bars are drawn for each treatment group

bacteria correlated with the enhanced mortality of infected larvae induced upon exposure to an identical dose of dexamethasone observed previously (Fig. 3).

To conclude, treatment of G. mellonella larvae with dexamethasone 21-phosphate enhanced the lethality induced by infection with $E$. coli or $K$. pneumoniae in a dose- and inoculum size-dependent manner. The enhanced lethality observed correlated with proliferation of bacteria in the larvae that could be attributed to dexamethasone inhibiting haemocyte phagocytosis and thus acting as an immunosuppressant.

The efficacy of antibiotic therapy versus K. pneumoniae or $E$. coli infection is reduced in larvae treated with dexamethasone 21-phosphate

The MICs of ceftazidime for the four strains used in this work were: E. coli NCTC12241 and NCTC13353, 0.25 and 

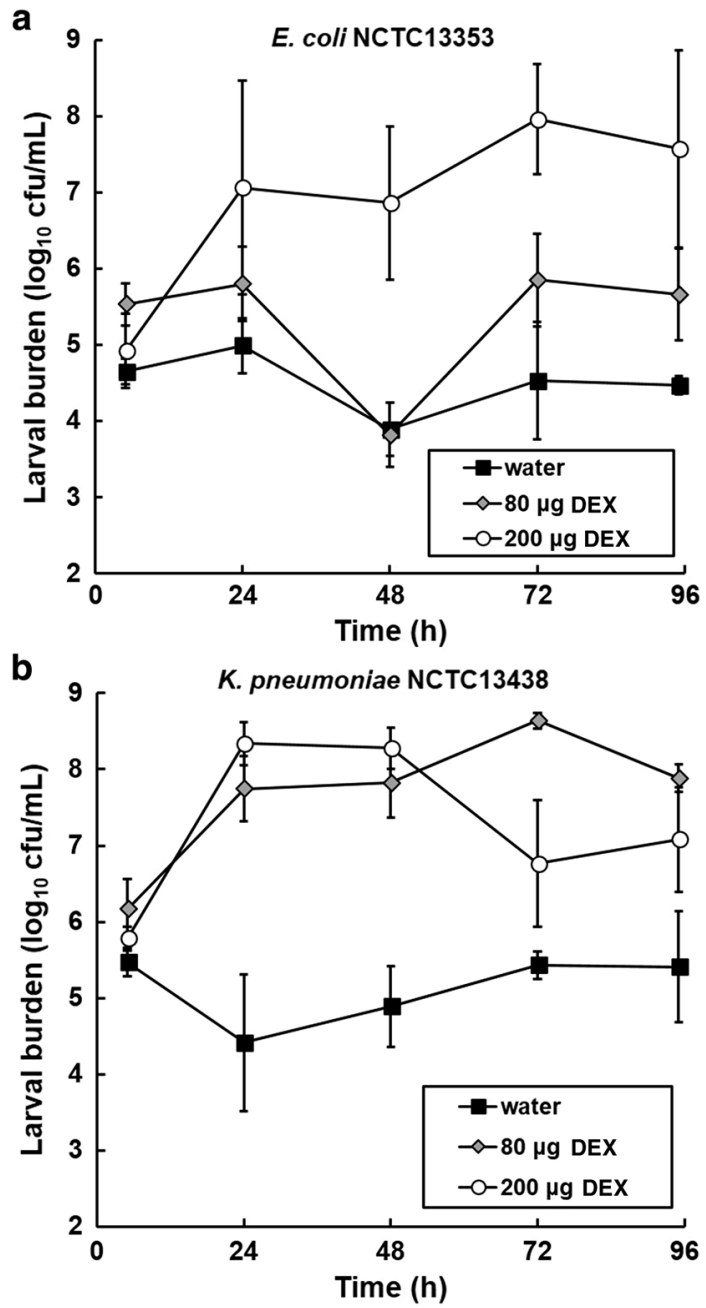

Fig. 5 Effect of pre-treatment with dexamethasone 21-phosphate (DEX; 80 or $200 \mu \mathrm{g}$ per larva) and subsequent infection with $1.0 \times 10^{6}$ cells per larva of $E$. coli NCTC13353 (a) or $5.6 \times 10^{4}$ cells per larva of K. pneumoniae NCTC13438 (b) on larval burden of bacteria during incubation at $37{ }^{\circ} \mathrm{C}$ for $96 \mathrm{~h}$. Larvae treated with dexamethasone 21-phosphate were compared to controls treated with sterile water. Dexamethasone 21-phosphate or water was administered to the larvae $10 \mathrm{~min}$ prior to infection with live bacteria. Larval burden was determined from 5 individual larvae every $24 \mathrm{~h}$. The data shown is from duplicate experiments and the error bars indicate the \pm standard error

$64 \mathrm{mg} / \mathrm{L}$, respectively; and K. pneumoniae NCTC9633 and NCTC13438, 0.5 and $\geq 512 \mathrm{mg} / \mathrm{L}$, respectively. The effect of treatment with ceftazidime, with or without prior exposure to dexamethasone-21-phosphate, on larvae infected with the two sensitive strains, E. coli NCTC12241 and $K$. pneumoniae NCTC9633, is shown in Fig. $6.2 \mathrm{~h}$ post-infection, the larvae were treated with either PBS or increasing doses of ceftazidime (10 or $25 \mathrm{mg} / \mathrm{kg}$ for $K$. pneumoniae NCTC9633; and 1 or $2 \mathrm{mg} / \mathrm{kg}$ for E. coli NCTC12241). Supporting the previous observations, in the control groups administered PBS rather than the antibiotic, exposure to dexamethasone 21-phosphate (200 $\mu \mathrm{g}$ per larva) prior to infection resulted in enhanced lethality of both strains compared to treatment with water. However, in control groups treated with water in the absence of dexamethasone 21-phosphate, administration of ceftazidime significantly improved survival of larvae, infected with either strain, in a dose-dependent manner. In comparison, after prior exposure to dexamethasone 21-phosphate, the efficacy of identical doses of ceftazidime was significantly reduced.

In summary, prior exposure to the immunosuppressant dexamethasone 21-phosphate not only enhances G. mellonella lethality induced by infection with $E$. coli or $K$. pneumoniae but also reduces the efficacy of a normally effective antibiotic treatment.

\section{Discussion}

The sensitivity of G. mellonella larvae to infection by the E. coli or K. pneumoniae strains in this work compares favourably with that identified in previous studies [8-11, 13-15]. For example, between $1.0 \times 10^{5}$ and $5.0 \times 10^{6}$ CFU per larva are required for $E$. coli to induce significant mortality [13-15] and between $1.0 \times 10^{5}$ and $1.0 \times 10^{6}$ CFU per larva for $K$. pneumoniae [8-11]. In contrast, different strains of Pseudomonas aeruginosa induced significant lethality of G. mellonella after infection with as few as 25 cells per larva $[16,17]$. This implies that either the innate immune system of $G$. mellonella is better adapted to combat $K$. pneumoniae and $E$. coli, or these pathogens are less virulent than $P$. aeruginosa in the larvae, or a combination of both factors could contribute to the observed differences.

In response to bacterial infection, the immune system of G. mellonella forms melanotic nodules whose formation is regulated by signalling molecules termed eicosanoids. Nodules form when insect haemocytes degranulate upon contact with bacteria followed by activation of the prophenoloxidase cascade which results in the formation of melanin around the bacteria. Subsequently, a capsule forms around the sequestered bacteria in the developing nodule due to attachment and spreading of further haemocytes followed by the commencement of phagocytic activity to remove and destroy the bacteria [21]. Mandato et al. [20] showed that the eicosanoid biosynthesis inhibitor dexamethasone inhibited prophenoloxidase activation in vivo and haemocyte spreading and phagocytosis in vitro. Supporting this, a recent study showed that administration of dexamethasone repressed G. mellonella innate immunity in a similar fashion to injected entomopathogenic nematode cuticles [22].

This work has shown that the anti-inflammatory drug dexamethasone 21-phosphate also acts as an 
Fig. 6 Effect of ceftazidime treatment on survival of $G$. mellonella larvae pre-exposed to dexamethasone 21-phosphate (DEX; $200 \mu \mathrm{g}$ per larva) prior to infection with $2.9 \times 10^{5}$ cells per larva of $K$. pneumoniae NCTC9633 (a, b), or $2.5 \times 10^{4}$ cells per larva of $E$. coli $\mathrm{NCTC} 12241$ (c, d). E. coli NCTC12241 and $K$. pneumoniae NCTC9633 do not possess $\beta$-lactamases and were sensitive to ceftazidime in vitro with MIC values of 0.25 and $0.5 \mathrm{mg} / \mathrm{L}$, respectively. Survival was measured during incubation at $37^{\circ} \mathrm{C}$ for $96 \mathrm{~h}$, and dexamethasone 21-phosphate-treated groups $(\mathbf{b}, \mathbf{d})$ were compared to controls treated with water (a, c). Dexamethasone 21-phosphate or water was administered to the larvae $10 \mathrm{~min}$ prior to infection with live bacteria. A single dose of ceftazidime (CAZ) was administered $2 \mathrm{~h}$ post-infection: 10 and $25 \mathrm{mg} / \mathrm{kg}$ for $K$. pneumoniae NCTC9633 (a, b), and 1 and $2 \mathrm{mg} / \mathrm{kg}$ for E. coli $\mathrm{NCTC} 12241$ (c, d). Asterisk indicates a group with significantly enhanced survival compared to the infected control treated with PBS $(p<0.05, \log -$ rank test). $n=30$ (pooled from duplicate experiments)

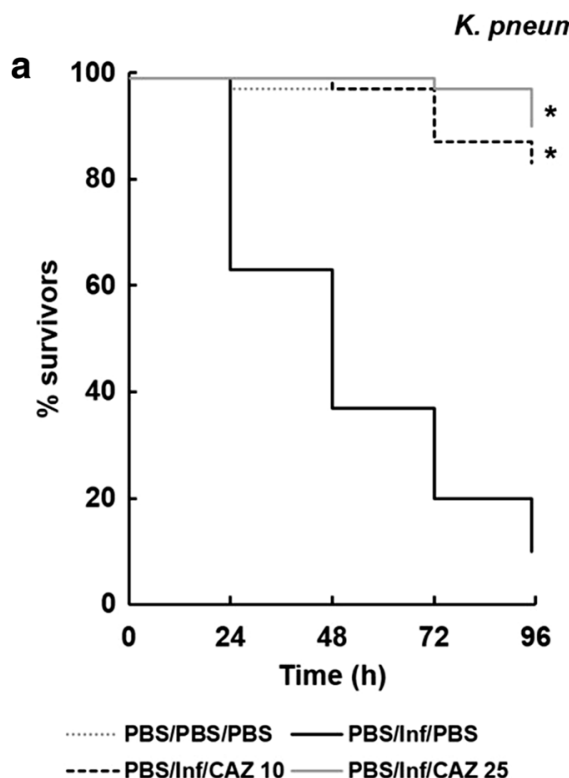

iae NCTC9633

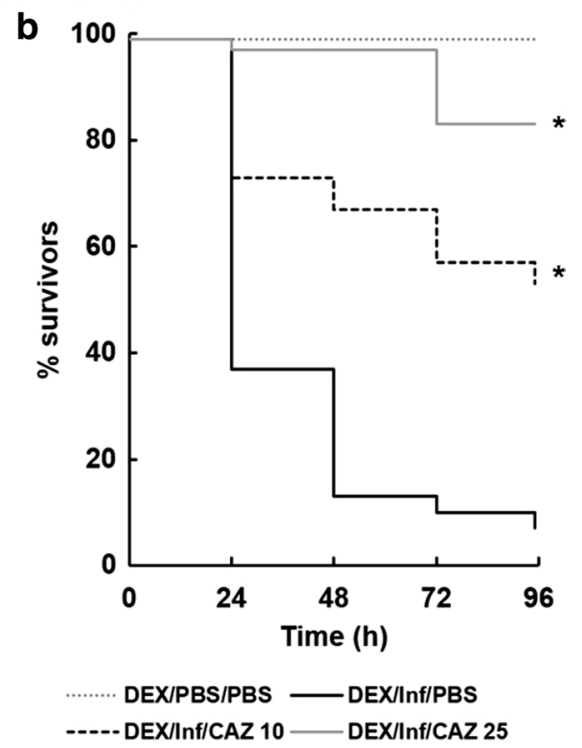

E. coli NCTC12241

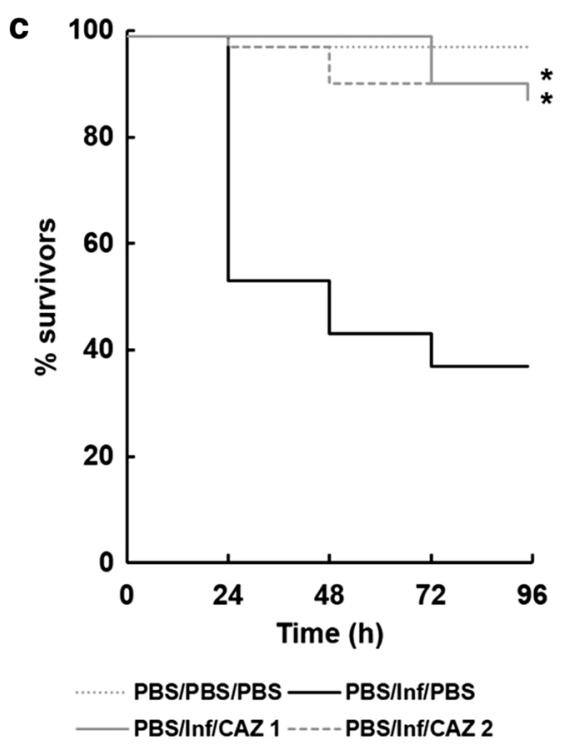

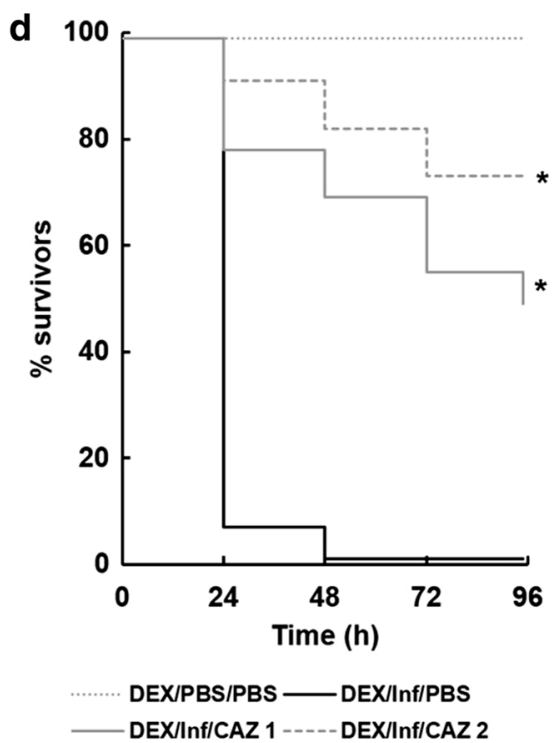

immunosuppressant in $G$. mellonella larvae. Larvae exposed to the drug become more sensitive to infection by $E$. coli and $K$. pneumoniae and die more rapidly than untreated equivalents. This enhanced lethality occurs due to the uncontrolled proliferation of bacteria within the infected larvae that can be at least partially attributed to the inhibitory effect treatment with the drug has on haemocyte phagocytosis. Supporting these findings, studies using the alternative immunosuppressant drugs, cyclosporin A [23] and hydrocortisone [24], also enhanced the lethality of bacterial infections, by Pseudomonas aeruginosa and Enterobacter cloacae, respectively, in G. mellonella larvae.

Dexamethasone 21-phosphate has the advantage of being water soluble and easier to administer then dexamethasone which is only soluble at use concentrations in organic solvents, such as ethanol or dimethyl sulphoxide, which, in our hands, had toxic side effects on G. mellonella larvae (data not shown).

Doses of dexamethasone phosphate used in humans are variable depending on the underlying condition and severity of disease. For sepsis, doses between 0.3 and $30 \mathrm{mg} /$ $\mathrm{kg}$ have been administered in a range of published trials (reviewed in [25]). The highest effective dose administered in this work (200 $\mu \mathrm{g}$ per larva) equates to a dose of $800 \mathrm{mg} / \mathrm{kg}$, indicating that the immunosuppressive effect of dexamethasone phosphate is far more potent in human patients than in G. mellonella larvae.

Successful application of an immunosuppressant with the $G$. mellonella infection model is significant because immunosuppression may facilitate studies on infection and 
treatment of alternative human pathogens that have low virulence or do not normally induce acute infections in this invertebrate [26]. Furthermore, the use of immunosuppression will better mimic the physiological state of many human patients that suffer from hospital-acquired infections caused by organisms such as E. coli and $K$. pneumoniae and thus improve the clinical relevance of this invertebrate model. Finally, this work has shown that immunosuppression can be used to improve the specificity of infection in G. mellonella. For example, as discussed earlier, infections with $E$. coli and $K$. pneumoniae require high inoculum numbers to induce lethality of the larvae. As a consequence of this, infections with identical inocula that were heat-killed induced high levels of larval mortality that could not be attributed solely to infection by live bacteria but were also due to the presence of toxic components of the dead bacteria. Treatment with dexamethasone 21-phosphate allowed infections with smaller inoculum sizes of both $E$. coli and $K$. pneumoniae to induce substantial larval lethality where equivalent heat-killed inocula did not induce significant pathogenicity. This implies that the specificity of infection of G. mellonella can be significantly improved by using dexamethasone phosphate to alleviate the need to use high cell number inocula of bacterial species such as $E$. coli and K. pneumoniae. A possible explanation for the observed toxicity of the dead cells may be due to the induction of a pro-inflammatory effect analogous to that seen with Gramnegative sepsis in humans, but this would require additional investigation that is outside the scope of this study.

Distinct from administering a specific drug to induce immunosuppression, but corroborating this finding, enhanced susceptibility of $G$. mellonella larvae to microbial infection has been reported after exposure to different stress factors that impair aspects of innate immunity. For example, infection by Candida albicans or Staphylococcus aureus induced greater larval mortality after prolonged incubation over a 10 -week period at $15{ }^{\circ} \mathrm{C}$ that was attributed to a combination of a decline in certain metabolic pathways and changes in the relative abundance of haemocytes [27]. Similarly, nutrient deprivation of larvae also has immunosuppressive effects and results in lower haemocyte density and a reduction in the expression of antimicrobial peptides leading to increased susceptibility to $C$. albicans infection [28].

In the clinical setting, the co-administration of antiinflammatory drugs with antibiotics remains controversial. Adjunctive treatment with corticosteroids such as dexamethasone is used to decrease inflammation and mortality in bacterial meningitis or sepsis [25, 29]. However, evidence has been presented that this could contribute to failure of antibiotic therapy [30, 31]. In this work, administration of ceftazidime to G. mellonella larvae infected with susceptible strains of E. coli and K. pneumoniae resulted in enhanced survival. However, the efficacy of the antibiotic was reduced in immunosuppressed larvae that had been treated with dexamethasone 21-phosphate prior to infection. This could be explained by the immunosuppressant drug interfering with the inhibitory action of the antibiotic, or because the immunosuppressant inhibits aspects of innate immunity, and as a consequence allows the infecting bacteria to proliferate (as shown in this study), an identical dose of antibiotic would not be as efficacious as it would be in larvae not immunosuppressed. Alternatively, dexamethasone can induce overexpression of $t o l C$, repression of OmpF, stimulate antibiotic efflux and ultimately reduce the susceptibility of $E$. coli to antibiotics in vitro [32].

The reduction in antibiotic efficacy seen with immunosuppressive therapy merits further investigation with the $G$. mellonella model to help clarify the relationship between the immune system and antibiotic treatment to clearance of bacteria and the relative contributions of each to abrogation of infection. Notably, this could have some clinical relevance to the adjunctive use of anti-inflammatory drugs with antibiotics that is used to tackle some serious bacterial infections.

To conclude, the demonstration of an effective immunosuppressant regimen in the G. mellonella infection model shown here should facilitate additional studies on novel treatments to combat MDR Gram -ve bacteria, such as $E$. coli and $K$. pneumoniae, that cause serious infections in hospitals caring for already severely ill patients and further develops G. mellonella as a serious alternative model host.

Acknowledgments MPT was the recipient of an ERASMUS training grant. FE is supported by the University of St Andrews.

\section{Compliance with ethical standards}

Conflict of interest The authors declare that they have no conflict of interest.

Open Access This article is distributed under the terms of the Creative Commons Attribution 4.0 International License (http://creativecommons.org/licenses/by/4.0/), which permits unrestricted use, distribution, and reproduction in any medium, provided you give appropriate credit to the original author(s) and the source, provide a link to the Creative Commons license, and indicate if changes were made.

\section{References}

1. Centers for Disease Control and Prevention (2013) Antibiotic resistance threats in the United States, 2013. Atlanta: CDC, 2013. http://www.cdc.gov/drugresistance/threat-report-2013/pdf/ ar-threats-2013-508.pdf.. Accessed 11 November 2015

2. Peleg A, Hooper D (2010) Hospital-acquired infections due to Gram-negative bacteria. New Eng J Med 362:1804-1813 
3. Morrill HJ, Pogue JM, Kaye KS, LaPlante KL (2015) Treatment options for carbapenem-resistant Enterobacteriaceae infections. Open Forum Infect Dis. doi:10.1093/ofid/ofv050

4. Akova M, Daikos GL, Tzouvelekis L, Carmeli Y (2012) Interventional strategies and current clinical experience with carbapenemase-producing Gram-negative bacteria. Clin Microbiol Infect 18:439-448

5. Woodford N, Zhang J, Warner M, Kaufmann ME, Matos J, MacDonald A, Brudney D, Sompolinsky D, Navon-Venezia S, Livermore DM (2008) Arrival of Klebsiella pneumoniae producing KPC carbapenemase in the United Kingdom. J Antimic Chemother 62:1261-1264

6. Woodford N, Ward ME, Kaufmann ME, Turton J, Fagan EJ, James D, Johnson AP, Pike R, Warner M, Cheasty T, Pearson A, Harry S, Leach JB, Loughrey A, Lowes JA, Warren RE, Livermore DM (2004) Community and hospital spread of Escherichia coli producing CTX-M extended-spectrum $\beta$-lactamases in the UK. J Antimic Chemother 54:735-743

7. Desbois A (2012) Coote P (2012) Utility of greater wax moth larva (Galleria mellonella) for evaluating the toxicity and efficacy of new antimicrobial agents. Adv Appl Microbiol 78:25-53

8. Insua JL, Llobet E, Moranta D, Pérez-Gutiérrez C, Tomás Garmendia J, Bengoechea JA (2013) Modeling Klebsiella pneumoniae pathogenesis by infection of the wax moth Galleria mellonella. Inf Immun 81:3552-3565

9. Wand ME, McCowen JWI, Nugent P, Sutton JM (2013) Complex interactions of Klebsiella pneumoniae with the host immune system in a Galleria mellonella infection model. J Med Microbiol 62:1790-1798

10. McLaughlin MM, Advincula MR, Malczynski M, Barajas G, Qi C, Scheetz MH (2014) Quantifying the clinical virulence of Klebsiella pneumoniae producing carbapenemase Klebsiella pneumoniae with a Galleria mellonella model and a pilot study to translate to patient outcomes. BMC Infect Dis 14:31

11. Benthall G, Touzel RE, Hind CK, Titball RW, Sutton JM, Thomas RJ, Wand ME (2015) Evaluation of antibiotic efficacy against infections caused by planktonic or biofilm cultures of Pseudomonas aeruginosa and Klebsiella pneumoniae in Galleria mellonella. Int J Antimic Agents 46:538-545

12. Alghoribi MF, Gibreel TM, Dodgson AR, Beatson SA, Upton M (2014) Galleria mellonella infection model demonstrates high lethality of ST69 and ST127 uropathogenic E. coli. PLoS ONE 9:e101547

13. Ciesielczuk H, Betts J, Phee L, Doumith M, Hope R, Woodford $\mathrm{N}$, Wareham DW (2015) Comparative virulence of urinary and bloodstream isolates of extra-intestinal pathogenic Escherichia coli in a Galleria mellonella model. Virulence 6:145-151

14. Leuko S, Raivio TL (2012) Mutations that impact the Enteropathogenic Escherichia coli Cpx envelope stress response attenuate virulence in Galleria mellonella. Inf Immun 80:3077-3085

15. Williamson DA, Mills G, Johnson JR, Porter S, Wiles S (2014) In vivo correlates of molecularly inferred virulence among extraintestinal pathogenic Escherichia coli (ExPEC) in the wax moth Galleria mellonella model system. Virulence 5:388-393

16. Jander G, Rahme L, Ausubel F (2000) Positive correlation between virulence of Pseudomonas aeruginosa mutants in mice and insects. J Bacteriol 182:3843-3845

17. Hill L, Veli N, Coote PJ (2014) Evaluation of Galleria mellonella larvae for measuring the efficacy and pharmacokinetics of antibiotic therapies against Pseudomonas aeruginosa infection. Int J Antimicrob Agents 43:254-261

18. Holm S (1979) A simple sequentially rejective multiple test procedure. Scand J Stat 6:65-70

19. Adamson DH, Krikstopaityte V, Coote PJ (2015) Enhanced efficacy of putative efflux pump inhibitor/antibiotic combination treatments versus MDR strains of Pseudomonas aeruginosa in a Galleria mellonella in vivo infection model. J Antimic Chemother 70:2271-2278

20. Mandato GA, Diehl-Jones WL, Moore SJ, Downer RGH (1997) The effect of eicosanoid biosynthesis inhibitors on prophenoloxidase activation, phagocytosis and cell spreading in Galleria mellonella. J Insect Physiol 43:1-8

21. Ratcliffe NA, Rowley AF (1979) Role of hemocytes in defence against biological agents. In: Gupta AP (ed) Insect hemocytes development, forms, functions and techniques. Cambridge University Press, Cambridge, pp 331-414

22. Yi Y, Wu G, Li M (2016) Eicosanoids mediate Galleria mellonella immune response to hemocoel injection of entomopathogenic nematode cuticles. Parasitol Res 115:597-608

23. Fiolka MJ (2008) Immunosuppressive effect of cyclosporin A on insect humoral immune response. J Invert Pathol 98:287-292

24. Jarosz J (1994) Hydrocortisone, a suppressive agent of inducible antibacterial immunity in Galleria mellonella (Insect: Lepidoptera). Cytobios 80:243-248

25. Volbeda M, Wetterslev J, Gluud C, Zijlstra JG, van der Horst ICC, Keus F (2015) Glucocorticosteroids for sepsis: systematic review with meta-analysis and trial sequential analysis. Intensive Care Med 41:1220-1234

26. Bergin D, Brennan M, Kavanagh K (2003) Fluctuations in haemocyte density and microbial load may be used as indicators of fungal pathogenicity in larvae of Galleria mellonella. Microb Infect 5:1389-1395

27. Browne N, Surlis C, Maher A, Gallagher C, Carolan JC, Clynes M, Kavanagh K (2015) Prolonged pre-incubation increases the susceptibility of Galleria mellonella larvae to bacterial and fungal infection. Virulence 6:458-465

28. Banville N, Browne N, Kavanagh K (2012) Effect of nutrient deprivation on the susceptibility of Galleria mellonella larvae to infection. Virulence 3:497-503

29. Borchorst S, Møller K (2012) The role of dexamethasone in the treatment of bacterial meningitis-a systematic review. Acta Anaesthesiol Scand 56:1210-1221

30. Thomas R, Le Tulzo Y, Bouget J, Camus C, Michelet C, Le Corre P, Bellissant E (1999) Trial of dexamethasone treatment for severe bacterial meningitis in adults. Adult Meningitis Steroid Group. Intensive Care Med 25:475-480

31. Levy MM, Dellinger RP, Townsend SR, Linde-Zwirble WT, Marshall JC, Bion J, Schorr C, Artigas A, Ramsay G, Beale R, Parker MM, Gerlach H, Reinhart K, Silva E, Harvey M, Regan S, Angus DC (2010) The Surviving Sepsis Campaign: results of an international guideline-based performance improvement program targeting severe sepsis. Intensive Care Med 36:222-231

32. Tavío MM, Aquili VD, Poveda JB (2014) Four anti-inflammatory drugs induce tolC gene overexpression and repression of OmpF synthesis, enhance active efflux and decrease susceptibility to antibiotics in Escherichia coli AG100 strain. APMIS 122:1153-1155 